\title{
SELF-SUPERVISED LEARNING FOR MONOCULAR DEPTH ESTIMATION FROM AERIAL IMAGERY
}

\author{
M. Hermann ${ }^{\text {a,b,c }}$, B. Ruf ${ }^{\text {a,b,c* } *}$, M. Weinmann ${ }^{\text {b }}$, S. Hinz ${ }^{\text {b }}$ \\ ${ }^{\mathrm{a}}$ Fraunhofer IOSB, Karlsruhe, Germany - \\ \{max.hermann, boitumelo.ruf\}@iosb.fraunhofer.de \\ ${ }^{\mathrm{b}}$ Institute of Photogrammetry and Remote Sensing, KIT, Karlsruhe, Germany - \\ \{max.hermann, boitumelo.ruf, martin.weinmann, stefan.hinz\}@ kit.edu \\ ${ }^{\mathrm{c}}$ Fraunhofer Center for Machine Learning
}

\section{Commission II, WG 4, ICWG I/II}

KEY WORDS: Monocular Depth Estimation, Self-Supervised Learning, Deep Learning, Convolutional Neural Networks, SelfImproving, Online Processing, Oblique Aerial Imagery

\begin{abstract}
:
Supervised learning based methods for monocular depth estimation usually require large amounts of extensively annotated training data. In the case of aerial imagery, this ground truth is particularly difficult to acquire. Therefore, in this paper, we present a method for self-supervised learning for monocular depth estimation from aerial imagery that does not require annotated training data. For this, we only use an image sequence from a single moving camera and learn to simultaneously estimate depth and pose information. By sharing the weights between pose and depth estimation, we achieve a relatively small model, which favors real-time application. We evaluate our approach on three diverse datasets and compare the results to conventional methods that estimate depth maps based on multi-view geometry. We achieve an accuracy $\delta_{1.25}$ of up to $93.5 \%$. In addition, we have paid particular attention to the generalization of a trained model to unknown data and the self-improving capabilities of our approach. We conclude that, even though the results of monocular depth estimation are inferior to those achieved by conventional methods, they are well suited to provide a good initialization for methods that rely on image matching or to provide estimates in regions where image matching fails, e.g. occluded or texture-less regions.
\end{abstract}

\section{INTRODUCTION}

Dense depth estimation is one of the most important and intensively studied tasks in photogrammetric computer vision. It produces dense depth maps that contain depth estimates at each pixel and represent the 3D scene geometry from certain viewpoints. Depth maps are a key input to numerous applications, such as dense 3D reconstruction and model generation, navigation of autonomous vehicles such as robots, cars and unmanned aerial vehicles (UAVs), as well as scene interpretation and analysis. Given two or more images, that depict the same scene from different viewpoints, the process of dense depth estimation can be formulated as the problem of finding a dense correspondence field between the input images, which in turn can be transformed into a depth map, if the corresponding camera projection matrices are known. In this, it is assumed that the scene geometry does not change between the different images, which does not always hold, especially when trying to reconstruct a scene with dynamic objects, e.g. an urban area with moving cars or pedestrians, by means of Structure-from-Motion (SfM).

Recent advancements in the field of deep learning have led to an increasing effort in attempting to learn how to hypothesize a depth image from a single input image (Eigen et al., 2014; Godard et al., 2017; Li et al., 2015). Figure 1 illustrates the general approach for this task. This process, known as Monocular Depth Estimation, is motivated by the capabilities of humans to guess depth estimates from a single image of a known scene. Similar to the empirical knowledge that humans establish throughout their lifetime, state-of-the-art convolutional

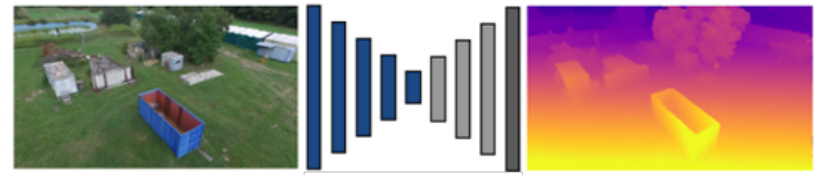

Figure 1. Schematic illustration of deep learning based monocular depth estimation.

neural networks (CNNs) are able to efficiently learn discriminative image cues that allow them to infer depth information from a new, so far unseen, image. Evidently, this only holds if the scene depicted in the new image is the same or at least similar to the scene that is covered by the training data.

There are a number of advantages to using monocular depth estimation instead of conventional image matching. For one, SfM is unstable when the camera is moving in the direction of the optical axis or if the camera has a narrow field-of-view. This is also the reason why image-based depth estimation in the context of autonomous driving is solely relying on a stereo camera setup. However, since the maximum depth range depends on the baseline between the images, a stereo camera is not always an option. Furthermore, monocular depth estimation does not suffer from occlusions, which are apparent to image matching in urban environments or when using UAVs flying at low altitudes. Finally, one major advantage is the processing speed which can be achieved by monocular methods. Since they only require a single image to estimate the scene depth, they can operate at a much higher frequency than SfM-based methods, which need at least two images.

* Corresponding author 
So far, a majority of studies (Eigen et al., 2014; Laina et al., 2016; Li et al., 2015) have focused on a supervised training of CNNs for the task of monocular depth estimation. They show that, with appropriate training data, state-of-the-art networks can even outperform conventional methods that rely on image matching. However, in many cases, supervised training is not feasible, first and foremost because it requires an appropriate dataset that contains labeled ground truth data. In the case of monocular depth estimation, this would mean to have a dataset with ground truth depth maps for each input image that is used for training. Acquiring such a dataset is cumbersome and costly. Thus, to the best of our knowledge, there only exist a few datasets (Geiger et al., 2012; Schöps et al., 2017; Silberman et al., 2012) that provide suitable data to allow for a supervised learning for monocular depth estimation. However, none of them is appropriate to learn to predict a depth map from a single aerial image, since they all consist of terrestrial imagery.

To overcome the limitation of such datasets, recent work on Self-supervised Monocular Depth Estimation (SMDE) has proven that it is also possible to train a network which is designed for monocular depth estimation by only using image pairs from a stereo camera or a video from a single moving camera. During self-supervised training, depth estimation is posed as a view synthesis and image reconstruction problem in which one image is transformed into the view of another camera given the predicted depth and matched with the real image of the second camera. A network has learned to predict the depth correctly, if the transformed image corresponds to the actual image taken by the second camera. The key advantage of the SMDE approach is that it does not require any special training data, and thus eliminates a major impediment of deep learning based approaches. This allows SMDE to be applied to any domain without the need for a costly acquisition of an appropriate training dataset.

Based on the results and advancements achieved by SMDE in the context of autonomous driving and driver assistance, we adopt the approach in our work to learn monocular depth estimation from aerial imagery captured by a single camera mounted to a commercial off-the-shelf UAV. Thus, in this work

- we show that it is possible to train a network in a selfsupervised manner to predict a depth map from a single aerial image, not relying on any labeled ground truth during training,

- we compare the results achieved by our SMDE approach to results obtained by conventional methods that estimate depth maps based on multi-view geometry, and we argue why it can be feasible to rely on SMDE instead,

- we evaluate the overall performance of the trained model and draw a conclusion about how well it can be generalized to unknown scenes.

We briefly review different approaches on supervised and selfsupervised monocular depth estimation in Section 2. This is followed by a presentation of our methodology in Section 3. In this, we give a detailed description on the self-supervised training for monocular depth estimation. We evaluate our approach on three different datasets and present the results in Section 4, together with a discussion, an ablation study and a comparison to traditional methods. Furthermore, we provide a discussion on generalization and the self-improving capabilities of the presented approach. Finally, we provide a brief summary, concluding remarks and a short outlook on future work in Section 5.

\section{RELATED WORK}

Approaches that allow for dense depth estimation from aerial imagery often rely on Markov Random Fields (MRFs). In this, an energy functional is formulated and minimized to compute a smooth dense depth map while preserving depth continuities and geometric relationships, such as occlusions. In particular, the so-called Semi-Global Matching (SGM) (Hirschmueller, 2008), which minimizes an energy functional by employing dynamic programming, has proven to be well suited for dense image matching from aerial imagery, due to its trade-off between accuracy and efficiency (Rothermel et al., 2012; Ruf et al., 2019; Wenzel et al., 2013). SGM can be applied to images from a stereo camera setup as well as to images acquired from a single moving camera. If the latter is the case, information about the movement of the camera is required.

\subsection{Supervised learning}

Besides classic approaches, there are also learning-based approaches to dense depth estimation. Most of them are based on $\mathrm{CNNs}$, which are trained in a supervised manner using ground truth depth maps (Eigen et al., 2014; Laina et al., 2016; Li et al., 2015). However, gathering sufficient amounts of training data is costly, so the number of available datasets is small. Especially in case of aerial imagery, we are not aware of any dataset that contains enough color images and dense depth maps to perform supervised training on a large scale. Even though some studies suggest to use synthetic datasets for training (JohnsonRoberson et al., 2016; Mayer et al., 2018), it is still very timeconsuming to generate large amounts of realistic data. Moreover, the use of a trained model across different domains, e.g. transferring between terrestrial and aerial imagery, or training a model on a synthetic dataset and using it to real-world data, is still an open issue and doesn't always provide satisfying results.

\subsection{Self-supervised learning}

A possible solution to not being dependent on a dataset with ground truth depth maps are self-supervised techniques. Instead of comparing the predicted depth map with a ground truth, the problem is modeled as the problem of novel view synthesis. In earlier works, this approach was used to generate new viewpoints from given imagery by estimating the depth of the scene (Flynn et al., 2016; Xie et al., 2016). In this, the depth map is only used as an auxiliary resource, since an understanding of the scene geometry is necessary to sample correct images. But there are also approaches that take advantage of this in order to predict depth maps. Here, the process of view synthesis is only used as a training method by comparing the synthetic image with a given image from the corresponding viewpoint. This is possible either by estimating the depth from several images as shown by Khot et al. (2019), or by estimating the depth from only one image. For the process of sampling synthetic images, the extrinsic parameters between individual input images as well as the intrinsic parameters of the cameras must be known. This is why methods based on stereo cameras like the one proposed by Godard et al. (2017) are particularly suitable, since rotation and translation between left and right images are fixed and can be calibrated a priori. However, relying on a stereo camera setup is not always feasible. Consequently, there are methods that exploit the SfM paradigm during training and rely on images from only one camera by additionally estimating the relative transformation between the images in order to estimate the depth (Mahjourian et al., 2018; Wang et al., 2018; Zhou et al., 2017). This allows to learn directly from a video without the need for additional ground truth, making it much 
easier to obtain suitable training material. It is especially suitable for monocular depth estimation from aerial imagery captured by commercial off-the-shelf UAVs.

\subsection{Self-supervised learning from aerial imagery}

Most of the techniques described above derive from the context of autonomous driving. Aerial imagery, however, is very different from street recordings, since not only the flight altitude varies, but also the angles and movement of the camera. Knöbelreiter et al. (2018) show that a self-supervised approach can also be applied to aerial photographs. They rely on rectified stereo image pairs and only use the predicted depth maps as an initialization to a hybrid approach based on a CNN and Conditional Random Fields (CRFs) (Knöbelreiter et al., 2017).

In contrast, we directly evaluate the predicted depth maps of our Self-supervised Monocular Depth Estimation and do not rely on a secondary procedure to refine the results. Furthermore, we only use the pictures of one camera as training material. The training material is obtained from videos captured by a UAV. Our approach is somewhat comparable to the ones presented in the context of autonomous driving, since both depth and camera movements are estimated by our model.

\section{METHODOLOGY}

In the following, we present and discuss our methodology for Self-supervised Monocular Depth Estimation ${ }^{1}$. Since we use a learning-based approach, we have structured our methodology with respect to training and inference, whereby we have additionally divided the section on the training phase into four subsections referring to depth and pose estimation as well as image projection and image reconstruction, i.e. loss calculation.

\subsection{Training}

The training data consists of image sequences depicting a scene for which the network is to be trained. From these input images, triplets are formed which serve as an input to the training process. Each triplet should show a static scene from three different viewpoints. In this, it is crucial that there exists enough parallax between the images to predict the depth, but not so much that it would hinder a matching between the images. In order to fulfill these conditions, the individual images of a triplet are sampled from the video with a certain offset. This offset is empirically determined for each dataset and depends, among other factors, on the flight speed and the frame rate of the video.

In the following, the middle image of a triplet is referred to as the reference image $I_{\text {ref }}$ and the other two as matching images $I_{\text {left }}, I_{\text {right }}$. In addition, the intrinsic camera parameters are required for the training procedure.

As shown in Figure 2, the training process is divided into four consecutive steps that are executed in each training iteration. 1) First, we perform monocular depth estimation to predict a depth map $D$ from $I_{\text {ref }}$. 2) Afterwards, the relative rotation and translation between the reference image $I_{\text {ref }}$ and the two adjacent images $I_{\text {left }}, I_{\text {right }}$ is estimated. 3) From these additional viewpoints, synthetic versions of the reference image are then sampled using the estimated depth map $D$ and relative camera poses $\mathbf{T}_{\mathrm{k}}=\left[\begin{array}{ll}\mathbf{R}_{k} & \mathrm{t}_{k}\end{array}\right]$. 4) In the last step, an image reconstruction error is calculated from these synthetic images and the original image. This error is backpropagated through the network, serving as the training loss.

\footnotetext{
1 Code available at: https://github.com/Max-Hermann/ SelfSupervisedAerialDepthEstimator/
}

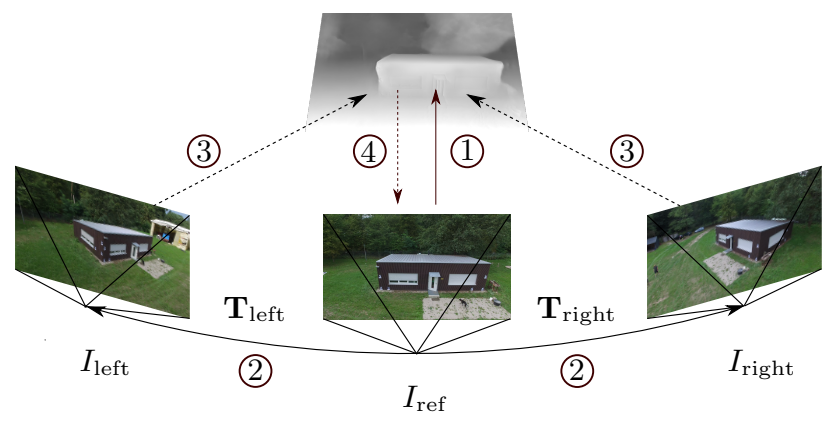

Figure 2. Training procedure with the estimation of depth (1) and pose (2) information, as well as the sampling of the synthetic images (3) and the calculation of the reconstruction error (4).

Evidently, the depth and pose estimates will be of low quality at the beginning of the training process, resulting in a poor image reconstruction. With each training iteration, the network will improve its capability to learn how to predict the corresponding information by minimizing the training loss. It is assumed, that the network has learned to predict the depth map and relative camera poses correctly, if the synthetic images generated from $I_{\text {left }}, I_{\text {right }}$ correspond to $I_{\text {ref }}$, i.e. when the reconstruction error is at its minimum.

3.1.1 Monocular depth estimation For monocular depth estimation, we use a network topology that is based on the UNet architecture developed by Ronneberger et al. (2015). It consists of an encoder and a decoder connected with multiple skip connections to include both high-level and low-level features. Based on our ablation study (cf. Section 4.5), the ResNet18 architecture (He et al., 2016) has shown to provide the best results when used as the encoder of the U-Net. As decoder, we adopt the approach of Godard et al. (2017), which uses nearest neighbor upsampling. In contrast to a transposed convolution (deconvolution), it does not require any additional parameters that need to be learned during training. The last layer of the U-Net uses a Sigmoid activation function to predict a depth map with the same dimensions as the input image.

3.1.2 Pose estimation Because we focus on real-time computation, a small size of our resulting model is important to us. To reduce the number of trainable parameters, which in turn increases the training speed, we share the weights of our depth and pose encoders. This means, that our network for pose estimation uses the same layers of the U-Net encoder used for depth estimation to compute feature maps from the input images. We feed all three images through the shared encoder and concatenate the resulting feature maps. These are then passed through three dense layers with subsequent global average pooling. In this way, the parameters of the relative rotation $\mathbf{R}$ and translation $t$ with respect to the reference image are estimated for each matching image. This approach is similar to the architecture shown in (Zhou et al., 2017). To sum up, although we differentiate between the networks for depth and pose estimation, both share a common encoder as depicted in Figure 3.

3.1.3 Image projection To learn the complex process of image projection and image sampling, and thus synthetic view generation, we use the concept of the Spatial Transformer Networks (STNs) presented by Jaderberg et al. (2015). STNs allow to learn only the parameters which are necessary for sampling instead of learning the whole algorithm. As sampling method, we use bilinear interpolation to generate synthetic images from a three-dimensional grid constructed from the predicted depth 


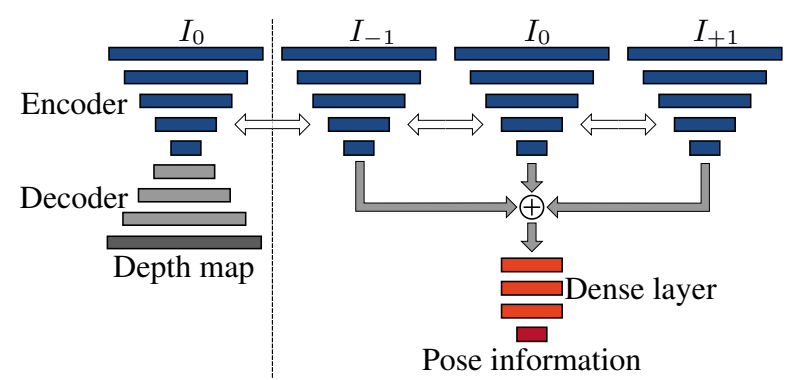

Figure 3. Network architecture with depth estimation (left) and pose estimation (right) networks, sharing the encoder (blue). For pose estimation, the feature maps are concatenated and passed through the dense layers with a final global average pooling.

maps. The required parameters are formalized below:

$$
I_{\mathrm{k} \rightarrow \mathrm{ref}}=I_{\mathrm{k}}\left[\operatorname{proj}\left(D, \mathbf{T}_{\mathrm{k}}, \mathbf{K}\right)\right]
$$

The projection $I_{\mathrm{k} \rightarrow \text { ref }}$ of the source image $I_{\mathrm{k}}$ onto the reference image $I_{\text {ref }}$ is formed by the sampling operator $[\cdot]$ from the pixel coordinates. In this context, $D$ is the predicted depth map of the reference image, $\mathbf{T}_{\mathrm{k}}$ is the transformation matrix into the camera coordinate system of the source image provided by the pose network and $\mathbf{K}$ is the intrinsic camera matrix.

For a more stable training, we use a multiscale estimation by outputting a depth map after each upsampling layer. To compare the resulting image projections with the reference image, the resolution of the depth map is adjusted. Instead of downsampling the source image to the resolution of the depth map, we use the approach presented by Godard et al. (2019) and upsample the depth maps to the resolution of the source image.

3.1.4 Image reconstruction and loss calculation In contrast to supervised learning methods, the self-supervised approach does not rely on a ground truth to which a loss function can be formulated in order to train the network. Thus, instead of comparing the predicted depth map with a ground truth depth map, we train the network to reconstruct the reference image from the matching images, given the predicted depth and relative transformation between the cameras. Accordingly, our training loss only indirectly allows reasoning about the quality of the depth maps, since it only measures the quality of the reconstructed images.

However, due to the structure of the networks, a good image reconstruction is only possible if depth and pose estimation provide accurate values. Therefore, the loss function is modeled to evaluate the quality of the image reconstruction. We additionally adjust the loss function to enforce smooth depth maps, which helps in weakly textured image regions. Thus, the aggregated loss $L$ is calculated as follows:

$$
L=L_{p}+\lambda L_{s},
$$

with $L_{p}$ being the photometric loss and $L_{s}$ being the smoothing loss weighted with the parameter $\lambda$. We empirically set the weighting factor to $\lambda=0.001$.

In order to account for occlusions, we use the pixel-wise minimum of the error maps from both matching images, since it can be assumed that areas in $I_{\text {ref }}$ which are occluded in $I_{\text {left }}$ will be visible in $I_{\text {right }}$, and vica versa (Kang et al., 2001). Therefore, the photometric loss $L_{p}$ is composed of the minimum image reconstruction error pe between the reference image $I_{\text {ref }}$ and the two sampled matching images $I_{k \rightarrow \mathrm{ref}}$ :

$$
L_{p}=\min _{I_{k}} p e\left(I_{\mathrm{ref}}, I_{k \rightarrow \mathrm{ref}}\right)
$$

Similar to (Godard et al., 2017; Mahjourian et al., 2018; Zhao et al., 2016), we employ a combined photometric loss consisting of a Structural Similarity (SSIM) and a L1 loss:

$$
p e\left(I_{a}, I_{b}\right)=\alpha \frac{1-S S I M\left(I_{a}, I_{b}\right)}{2}+(1-\alpha)\left\|I_{a}, I_{b}\right\|_{1}
$$

We use a value of $\alpha=0.15$, as this leads to a more stable convergence of our training.

To enforce smooth depth maps, we use an edge-aware smoothness loss like shown in (Wang et al., 2018). The underlying idea is that discontinuities in depth are accompanied by a change in the color gradient of the reference image. Because we encountered problems with degrading depth maps, we used the idea of depth normalization as presented in (Wang et al., 2018):

$$
L_{s}=\left|\partial_{x} d / \bar{d}\right| e^{-\left|\partial_{x} I_{\mathrm{ref}}\right|}+\left|\partial_{y} d / \bar{d}\right| e^{-\left|\partial_{y} I_{\mathrm{ref}}\right|}
$$

In this context, the gradient of the normalized depth map $d / \bar{d}$ is weighted with the color gradient of $I_{\mathrm{ref}}$ in $x$ and $y$ direction.

\subsection{Inference}

Since our approach aims for monocular depth estimation, the actual inference is performed on only one image. An arrangement of the images in groups of three is therefore no longer necessary here. Since image reconstruction using depth and pose information is only necessary for training, the network for estimating rotation and translation is no longer needed as well. Thus, during inference, we only use the network to predict the depth map from step one of our training procedure. This leads to a significant reduction in the parameters of the resulting model and, in turn, to an increase of the inference speed.

\section{EVALUATION}

In the following, we present and discuss the results achieved by our approach. First, we address the datasets and hyperparameters used. Then, we present the results achieved and compare them with results achieved by conventional methods. Additionally, we discuss the findings of our ablation study, done to evaluate different configurations of our model.

\subsection{Datasets}

In our experiments, we evaluate the overall performance of the Self-supervised Monocular Depth Estimation approach on three different datasets: two private real-world datasets and one synthetic one (cf. Figure 4). For the two real-world datasets, we generate ground truth data by employing state-ofthe-art SfM methods provided in the software bundle COLMAP (Schönberger and Frahm, 2016; Schönberger et al., 2016). Since the third dataset is synthetically generated, we can rely on the simulation capabilities of modern rendering engines to extract the precise ground truth data.

Our first dataset consists of video sequences showing three different rural scenes recorded from multiple different aerial viewpoints. In all sequences, the camera orbits around different objects of interest at different speeds, camera angles and altitudes. In this, the camera movement is only lateral. The second dataset contains multiple video sequences showing an ur- 
ban scene. In contrast to the first dataset, the camera movement here is only forwards with sharp turns inducing high amounts of motion blur. Additionally, the image quality and lighting is reduced due to bad weather conditions such as rain or mist. Both the rural and the urban dataset were captured with a DJI Phantom 3 Professional. For our evaluation, we extracted 5,000 images from each of the first two datasets and processed them with COLMAP to generate ground truth depth maps.

Since COLMAP does not produce perfectly accurate results, the ground truth depth maps still contain regions with errors. This causes apparent false positive and negative results that reduce the final score. To compensate for this issue, we synthetically generated a third dataset using the video game Grand Theft Auto V (GTA V). Synthetic data does not have the same level of detail, but it allows a manipulation of the environment. Thus, we simulated different kinds of flight trajectories, such as orbiting around buildings or flyovers with varying altitudes. Additionally, we randomized the weather conditions and time of day to create different scenes. To extract the depth maps from the video game, we used the software GTAVisionExport (Johnson-Roberson et al., 2016) and adapted it to our needs. In this way, we have collected 5,000 color images with corresponding high-resolution depth maps. We truncated these depth maps at a maximum of $100 \mathrm{~m}$ in order to provide comparable results to the other two datasets.

We train and evaluate our model on all three datasets simultaneously. This leads to the same results as training three separate models, showing that the model is capable to deal with diverse scenes and camera motions at the same time.

\subsection{Implementation details}

We implemented our model using the deep learning framework TensorFlow in version 1.15. To this end, we used a NVIDIA Titan X GPU for training and inference. We used the Adam optimizer with $\beta_{1}=0.9, \beta_{2}=0.999$ and a learning rate of 0.0002 . All results shown here are achieved by training with a batch size of 20. Smaller batch sizes can lead to unstable training, which is discussed in more detail in Section 4.5. In total, our model contains 21 million parameters at training time. Since the network for pose estimation can be omitted for inference, the model then only has a size of 17 million parameters.

The maximum training duration is difficult to determine, since training loss and evaluation error are decoupled. We have trained our networks from scratch up to a flattening of the training loss. By evaluation after each training period, we found out that the evaluation error does not improve after about one third of the total training time. We therefore approximate the maximum training time until the epoch that achieves the best result for the first time. With an image resolution of $384 \times 224$ pixels, our network needs about 100 epochs with a training duration of approx. 24 hours for the full dataset consisting of 15,000 images. The training time decreases proportionally with a reduction of the image resolution.

Additionally, we augmented the training data with randomly cropped and scaled copies of the input images. In order to avoid a bias in the camera movement, we flipped the images of a training triplet with a $50 \%$ probability. This reverses the motion that is to be estimated by the pose estimation network. Finally, we adjusted contrast, brightness, saturation and hue with random percentage deviations of up to $0.2,0.2,0.2$ and 0.1 .

Without optimizations, our model reaches speeds of more than $20 \mathrm{fps}$ at a resolution of $384 \times 224$ pixels during inference. This includes the pre-processing of images such as resizing, as well as loading them into the memory of the GPU and saving them to the hard drive. To measure the processing speed, the model was exported as a frozen graph with a batch size of 100 .

\subsection{Experimental results}

We quantitatively assess the results of the Self-supervised Monocular Depth Estimation approach with respect to the ground truth based on three metrics, namely the Root Mean Square Error (RMSE), the relative L1-Norm (L1-rel) as described in (Ruf et al., 2019) and the Accuracy. The latter is formulated in (Godard et al., 2019) as:

$$
\delta_{\theta}(d, \hat{d})=\frac{1}{m} \sum_{i=1}^{m} \max \left(\frac{d_{i}}{\hat{d}_{i}}, \frac{\hat{d}_{i}}{d_{i}}\right)<\theta
$$

Originating within the context of image-based classification, it renders a pixel within the estimated depth map as correct, if the estimate is within a certain threshold $\theta$ to the corresponding measurement in the ground truth depth map. Thus, $\delta_{1.25}$ describes the fraction of pixels $(m)$ of the ground truth depth map for which an estimate exists and for which the difference between the estimate $d$ and the ground truth $\hat{d}$ is not higher than $25 \%$ of $\hat{d}$. This measurement is also used by the KITTI (Menze and Geiger, 2015) and ETH3D (Schöps et al., 2017) benchmarks.

In our evaluation, the relative accuracy measures, i.e. L1-rel and $\delta_{\theta}$, are the most meaningful ones, since the different datasets differ greatly in the depicted scene depth and since the depth maps estimated by monocular depth estimation typically are free of a metric scale. To adjust the different resolution and depth range between an estimated depth map and the corresponding ground truth depth map, we have applied a simple image resizing and a median scaling. Furthermore, during evaluation, we use a 90/10 split of training and evaluation data.

\begin{tabular}{|l|c|c|c|c|}
\hline Dataset & RMSE & L1-rel & $\delta_{1.25}$ & $\delta_{1.05}$ \\
\hline Rural & 2.216 & 0.171 & 0.935 & 0.698 \\
Urban & 4.430 & 1.118 & 0.722 & 0.326 \\
Synthetic & 8.337 & 0.081 & 0.910 & 0.578 \\
\hline
\end{tabular}

Table 1. Quantitative results achieved by our approach. The values represent the mean score, averaged over all images in the corresponding dataset. The last two columns represent the accuracy at a threshold of $25 \%$ and $5 \%$ w.r.t. to the ground truth.

As shown in Table 1, our results vary greatly between the different datasets. The best accuracy is achieved on the rural dataset, followed by the synthetic dataset. The results achieved on the urban dataset are considerably worse, which we assume to be caused by the bad picture quality and the faulty ground truth.

Figure 4 shows examples of the predicted depth maps from each dataset, together with the corresponding reference image and the ground truth. In this, the depth is color coded, going from yellow (near) via red to blue (far). In the case of the two realworld datasets (rural and urban), the examples clearly show the errors in ground truth that have been induced by COLMAP.

Altogether, the results reveal that the presented self-supervised learning is capable of learning monocular depth estimation from aerial imagery. The quality of the resulting depth maps, in both quantitative and qualitative evaluation, is high with respect to the ground truth. Even though the model is incapable of predicting fine structures like vegetation and sharp edges, the essential scene geometry and objects are predicted correctly as can be derived from Figure 4. 

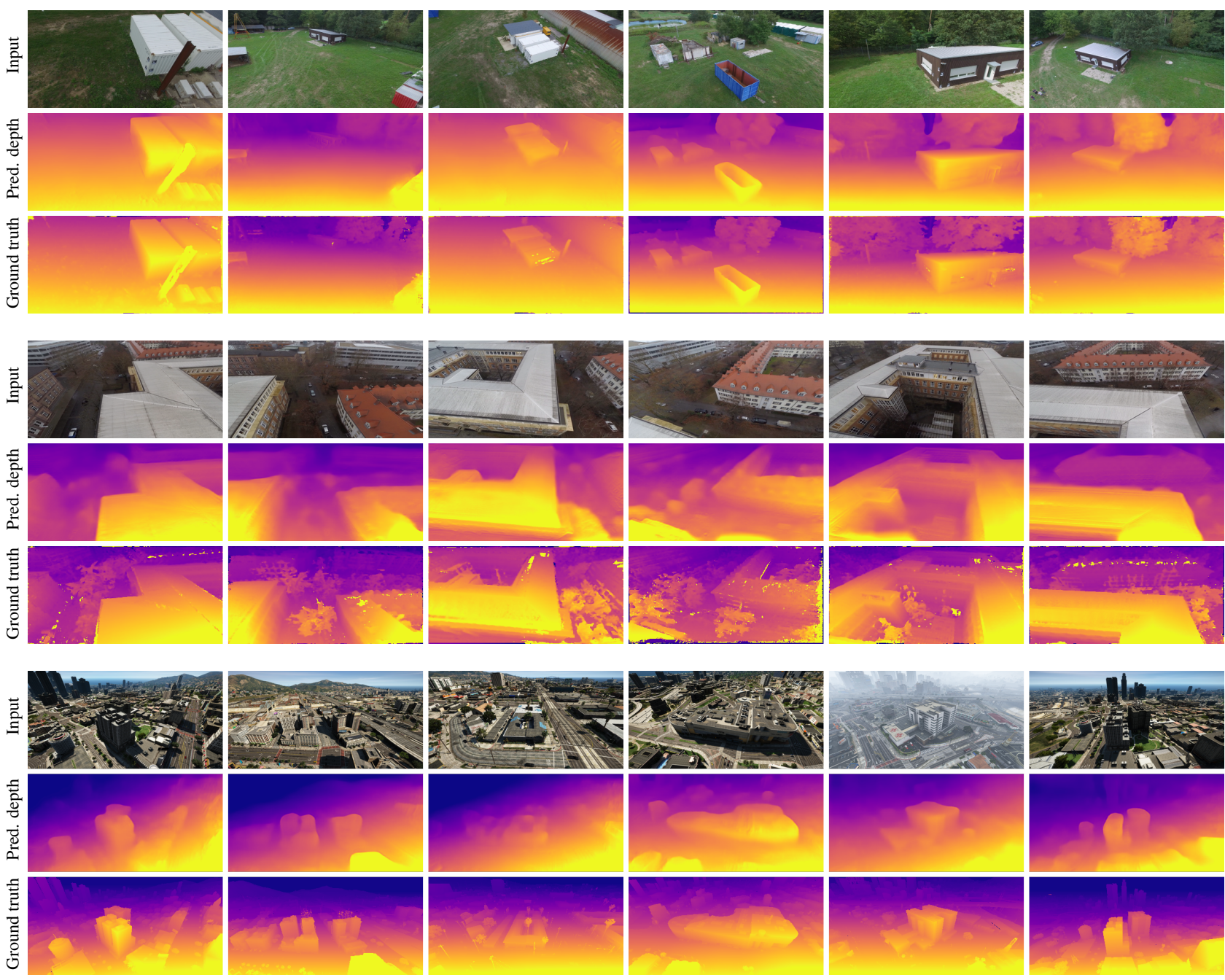

Figure 4. Qualitative results on all three datasets - from top to bottom: rural, urban and synthetic. The first row of each bundle shows the reference image for which the depth map is predicted. The second row depicts the depth map that is predicted by the presented SMDE approach. In the last row, the corresponding ground truth depth map is shown. In the case of the two real-world datasets, this is calculated by means of COLMAP.

\subsection{Comparison to traditional methods}

In addition to an evaluation of the presented Self-supervised Monocular Depth Estimation approach with respect to a ground truth, we have also compared the results to those achieved by conventional algorithms that are based on the SGM presented in (Hirschmueller, 2008). We have chosen SGM, since it is one of the most widely used algorithms for real-time depth estimation from two or more views. It allows to estimate accurate depth maps with reasonably low computational complexity.

For our comparison, we have selected the Semi-Global Block Matching (SGBM) variant provided in the computer vision library OpenCV, as well as the $\mathrm{SGM}^{s n}$ extension presented in (Ruf et al., 2019). The latter combines SGM with a multi-view plane-sweep sampling and incorporates local surface normals to improve the optimization. We have performed the comparison on the rural dataset, since it is the real-world dataset on which the best results are achieved by the SMDE. The results are listed in Table 2.

It is evident, that the results of the SMDE are inferior to those achieved by SGM. However, monocular depth estimation has a number of advantages compared to methods that rely on image matching. For one, it does not suffer from instabilities that

\begin{tabular}{|l|c|c|c|c|}
\hline Method & L1-rel & $\delta_{1.25}$ & $\delta_{1.15}$ & $\delta_{1.05}$ \\
\hline SMDE & 0.171 & 0.935 & 0.894 & 0.698 \\
SGBM & 0.052 & 0.986 & 0.876 & 0.767 \\
SGM $^{\text {sn }}$ & 0.042 & 0.968 & 0.951 & 0.890 \\
\hline
\end{tabular}

Table 2. Comparison of the presented SMDE approach to two variants of the SGM algorithm that perform depth estimation by epipolar geometry, namely SGM ${ }^{\text {sn }}$ from (Ruf et al., 2019) and SGBM from OpenCV.

are induced to SfM-based methods due to a bad choice of the camera movement. Furthermore, monocular methods produce fully dense depth maps, providing estimates in regions where image matching algorithms often fail, such as untextured or occluded areas. And finally, since the prediction of the depth map only requires a single input image, such methods can operate at a much higher frequency as they do not need to wait until the camera has moved to a new viewpoint.

\subsection{Ablation study}

Encoder topologies To study the impact of network topologies, we evaluated the use of three different architectures for the shared encoder as shown in Table 3. All three topologies, namely VGG-Net16 (Simonyan and Zisserman, 2014), ResNet18 (He et al., 2016) and DenseNet (Huang et al., 2017), 
are suitable for learning meaningful depth maps. However, ResNet18 achieves the best results, while simultaneously having less parameters than the other topologies and can thus be trained faster. We have also tried deeper variants like ResNet32 or ResNet50, but due to the limited graphics card memory, we had to reduce our batch size, resulting in very unstable training.

\begin{tabular}{|l|c|c|c|}
\hline Architecture & RMSE & L1-rel & $\delta_{1.25}$ \\
\hline VGG16 & 2.406 & 1.985 & $\mathbf{0 . 9 2 7}$ \\
ResNet18 & $\mathbf{2 . 1 9 1}$ & $\mathbf{0 . 7 3 4}$ & 0.926 \\
DenseNet & 2.548 & 5.370 & 0.912 \\
\hline
\end{tabular}

Table 3. Comparison of different network topologies used for the encoder.

Batch size During our final training, we have used a batch size of 20 , which was found empirically. We observe very unstable training if we used smaller batch sizes. In particular during early iterations, the training tends to diverge and get stuck in a local minimum. We assume this problem arises from the pose estimation network, since datasets with a complex camera movement, i.e. high amounts of rotation without forward motion, are especially affected. One possible explanation is that the encoder is shared between the depth estimation network and the pose estimation network. And since the pose estimation depends on depth information and vice versa, this could potentially create an endless loop in which the training does not converge. Using larger batch sizes increases the probability of processing different varieties of camera movements in one batch, and thus prevents divergence in early training iterations. After the network starts to predict reasonable depth maps, the batch size can be reduced. Furthermore, our experiments have shown, that batch sizes larger than 20 do not tend to produce better results but restrict rather the use of a higher input resolution due to the confined memory on the GPU.

Input resolution The resolution of the input and output data has a great influence on the training, as it is, depending on the batch size used, limited by the memory of the graphics card. Our results shown in Table 1 are achieved with an image resolution of $384 \times 224$ pixels. However, our model still produces good results when trained with a very low resolution of $192 \times 96$ pixels. Using the higher resolution increases the training time by a factor of four, achieving a quantitative improvement of only $2 \%$.

Size of image bundle Finally, to test whether our model benefits from more than three input images during training, we use sequences of five images, with four matching images projected onto the reference image. Our experiments show that this does not lead to superior results. We assume that the use of more matching images increases the probability of false positives in the process of image sampling, i.e. pixels that have a high similarity but are sampled from a wrong location, which hinders the training procedure.

\subsection{Generalization and self-improving capabilities}

In order to evaluate the capabilities of our approach to generalize to unknown data, we use a setup that contains two short video sequences without any overlap or common objects, but similar conditions, in terms of camera angle and weather. We begin by training a base model until convergence on the first sequence (Sequence $\mathrm{A}$ ) and then evaluate on the second one (Sequence B). As depicted in Figure 5, the accuracy $\delta_{1.25}$ drops by approx. $25 \%$ when switching to a new, yet similar sequence. From this, we conclude that the model generalizes rather moderately to unknown data, which hinders a practical use of the

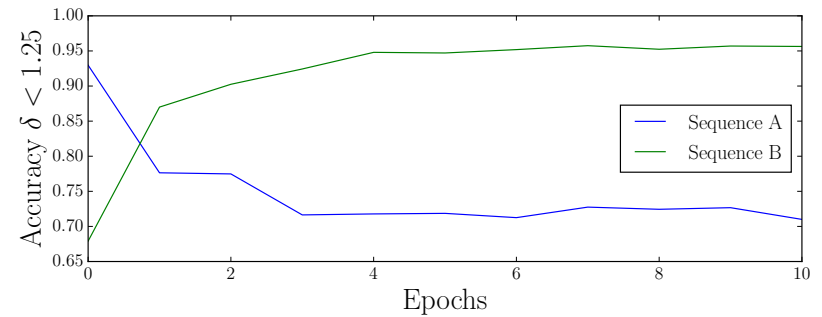

Figure 5. Evaluation of generalization and self-improving capabilities. After the network is trained until convergence on Sequence A, it is evaluated and fine-tuned on a new, yet similar Sequence B. The graph shows the accuracy achieved by the model on the two datasets during fine-tuning.

model. However, since it is a self-supervised method and does not require special ground truth data for training, the model can easily be retrained to learn the new sequence. This is often referred to as self-improving capabilities.

In our experiments, we evaluate how fast the model can be finetuned to the new data. To do so, we have not fixed the weights of our resulting model but continued training the network with the new Sequence B. As shown in Figure 5, the model can be fine-tuned within 2-4 epochs to a similar accuracy as initially achieved on Sequence A. One epoch corresponds to a training time of approx. 2 minutes with the same configuration as stated in Section 4.2. This fine-tuning is about 10 times faster than training from scratch, simultaneously achieving equal results.

Yet, the experiments also show that the network tends to forget the old sequence, as the accuracy achieved on Sequence A decreased with each epoch in which the model is fine-tuned on Sequence B. To prevent this, we also employed in another experiment a combined fine-tuning with images of both sequences, leading to the same increase of the accuracy on Sequence B, while maintaining the accuracy on Sequence A. This, however, again increases the training time, since the dataset is bigger.

Depending on the application, it should be decided whether it is more important to remember the old scene or to achieve a quick fine-tuning. In both cases, a base model that is as diverse as possible should be advantageous and can fine-tuned for a specialized use case. However, the method of self-improvement has also some disadvantages. In contrast to a standard application, in which only the depth estimation network is needed, the process of self-improvement also needs the network for pose estimation, increasing the size of the resulting model. Furthermore, not only the forward pass through the network is to be processed, as done during inference, but also the backpropagation in order to adjust the weights during the training, which increases the processing time for each input image.

\section{CONCLUSION \& FUTURE WORK}

To summarize, we show that it is possible to flexibly train a deep convolutional neural network to perform monocular depth estimation from aerial imagery. Our method relies on a selfsupervised learning procedure that does not require any special training data and thus can be applied to any image sequences from a video that was captured from a static scene with a moving camera. During training, our networks learn to predict depth information from a single input image as well as relative camera poses between three images. During inference, our approach only requires the network for depth estimation, decreasing the size of the resulting model and the processing time. We reduce the amount of trainable parameters by sharing the weights 
between the different encoders and by using simple upscaling instead of deconvolution, which makes our model also suitable for the use on systems with reduced hardware capabilities.

The conducted evaluation suggests a direct utilization of the estimated depth maps for a number of applications, such as realtime 3D modeling, navigation or scene interpretation without any further refinement. Even though the quality of the obtained depth maps is evidently inferior to the results achieved by conventional methods based on image matching, e.g. Semi-Global Matching, monocular depth estimation can operate at a higher frequency and does not suffer from typical drawbacks of SfM, such as occlusions or instabilities associated with inappropriate camera trajectories. In conclusion, Self-supervised Monocular Depth Estimation is well suited to complement conventional methods, for example by providing a good initialization or by providing estimates in regions where image matching fails, e.g. occluded or texture-less regions.

Furthermore, our experiments on practical use show that a trained model generalizes rather poorly and that an application of a trained model to a new dataset causes a significant drop in accuracy. However, by exploiting the self-improvement capability of our approach, we were able to show that a model can quickly be fine-tuned to the new data. Since we have only been using offline learning so far, an investigation on whether the self-improving capabilities of our model can also be realized with online learning is still to be done.

Additionally, a generalization to changes applied to data which has been seen during training, such as different weather or lighting conditions, as well modifications in the geometry of familiar scenes, is to be evaluated. This is extremely relevant for the use case of repeated flyovers, needed for the application of change detection or for the extension and update of existing 3D models.

Finally, the fact that the depth maps of monocular depth estimation methods do not have a metric scale makes it difficult to use them for accurate distance measures. Possible solutions to this could be the augmentation of the training data with a small number of metric depth maps or the use of a calibrated pose estimation method. Possible approaches would be the separation of depth and pose network, as well as the use of classical approaches of image processing, which would also help to handle more complex camera movements. However, an integration of conventional methods would reduce the benefit of an end-toend solution and the advantage of high processing rates.

\section{REFERENCES}

Eigen, D., Puhrsch, C. and Fergus, R., 2014. Depth map prediction from a single image using a multi-scale deep network. Proc. Advances in Neural Information Processing Systems, 2366-2374.

Flynn, J., Neulander, I., Philbin, J. and Snavely, N., 2016. DeepStereo: Learning to predict new views from the world's imagery. Proc. IEEE Conf. on Computer Vision and Pattern Recognition, 5515-5524.

Geiger, A., Lenz, P. and Urtasun, R., 2012. Are we ready for autonomous driving? The KITTI vision benchmark suite. Proc. IEEE Conf. on Computer Vision and Pattern Recognition, 3354-3361.

Godard, C., Mac Aodha, O. and Brostow, G. J., 2017. Unsupervised monocular depth estimation with left-right consistency. Proc. IEEE Conf. on Computer Vision and Pattern Recognition, 270-279.

Godard, C., Mac Aodha, O., Firman, M. and Brostow, G. J., 2019. Digging into self-supervised monocular depth estimation. Proc. IEEE International Conference on Computer Vision, 3828-3838.

He, K., Zhang, X., Ren, S. and Sun, J., 2016. Deep residual learning for image recognition. Proc. IEEE Conf. on Computer Vision and Pattern Recognition, 770-778.

Hirschmueller, H., 2008. Stereo processing by semiglobal matching and mutual information. IEEE Transactions on Pattern Analysis and Machine Intelligence, 30(2), 328-341.
Huang, G., Liu, Z., Van Der Maaten, L. and Weinberger, K. Q., 2017. Densely connected convolutional networks. Proc. IEEE Conf. on Computer Vision and Pattern Recognition, 4700-4708.

Jaderberg, M., Simonyan, K., Zisserman, A. and Kavukcuoglu, K., 2015. Spatial transformer networks. Proc. Advances in Neural Information Processing Systems, 2017-2025.

Johnson-Roberson, M., Barto, C., Mehta, R., Sridhar, S. N., Rosaen, K. and Vasudevan, R., 2016. Driving in the matrix: Can virtual worlds replace human-generated annotations for real world tasks? arXiv preprint arXiv: 1610.01983.

Kang, S. B., Szeliski, R. and Chai, J., 2001. Handling occlusions in dense multi-view stereo. Proc. IEEE Conf. on Computer Vision and Pattern Recognition, 103-110.

Khot, T., Agrawal, S., Tulsiani, S., Mertz, C., Lucey, S. and Hebert, M., 2019. Learning Unsupervised Multi-View Stereopsis via Robust Photometric Consistency. arXiv preprint arxiv:1905.02706.

Knöbelreiter, P., Reinbacher, C., Shekhovtsov, A. and Pock, T., 2017. End-to-end training of hybrid CNN-CRF models for stereo. Proc. IEEE Conf. on Computer Vision and Pattern Recognition, 2339-2348.

Knöbelreiter, P., Vogel, C. and Pock, T., 2018. Self-supervised learning for stereo reconstruction on aerial images. Proc. IEEE Intern. Geoscience and Remote Sensing Symposium, 4379-4382.

Laina, I., Rupprecht, C., Belagiannis, V., Tombari, F. and Navab, N., 2016. Deeper depth prediction with fully convolutional residual networks. Proc. IEEE International Conf. on 3D Vision, 239-248.

Li, B., Shen, C., Dai, Y., Van Den Hengel, A. and He, M., 2015. Depth and surface normal estimation from monocular images using regression on deep features and hierarchical CRFs. Proc. IEEE Conf. on Computer Vision and Pattern Recognition, 1119-1127.

Mahjourian, R., Wicke, M. and Angelova, A., 2018. Unsupervised learning of depth and ego-motion from monocular video using $3 \mathrm{~d}$ geometric constraints. Proc. IEEE Conf. on Computer Vision and Pattern Recognition, 5667-5675.

Mayer, N., Ilg, E., Fischer, P., Hazirbas, C., Cremers, D., Dosovitskiy, A. and Brox, T., 2018. What makes good synthetic training data for learning disparity and optical flow estimation? International Journal of Computer Vision, 126(9), 942-960.

Menze, M. and Geiger, A., 2015. Object scene flow for autonomous vehicles. Proc. IEEE Conf. on Computer Vision and Pattern Recognition, 3061-3070.

Ronneberger, O., Fischer, P. and Brox, T., 2015. U-Net: Convolutional networks for biomedical image segmentation. Proc. International Conf. on Medical Image Computing and Computer-Assisted Intervention, 234-241.

Rothermel, M., Wenzel, K., Fritsch, D. and Haala, N., 2012. SURE: Photogrammetric surface reconstruction from imagery. Proc. LowCost $3 D$ Workshop, Berlin, Germany, 1-9.

Ruf, B., Pollok, T. and Weinmann, M., 2019. Efficient Surface-Aware Semi-Global Matching with Multi-View Plane-Sweep Sampling. IS PRS Annals of the Photogrammetry, Remote Sensing and Spatial Information Sciences, IV-2/W7, 137-144.

Schönberger, J. L. and Frahm, J.-M., 2016. Structure-from-motion revisited. Proc. IEEE Conf. on Computer Vision and Pattern Recognition, 4104-4113.

Schönberger, J. L., Zheng, E., Frahm, J.-M. and Pollefeys, M., 2016. Pixelwise view selection for unstructured multi-view stereo. Proc. Europ. Conf. on Computer Vision, 501-518.

Schöps, T., Schönberger, J., Galliani, S., Sattler, T., Schindler, K., Pollefeys, M. and Geiger, A., 2017. A multi-view stereo benchmark with high-resolution images and multi-camera videos. Proc. IEEE Conf. on Computer Vision and Pattern Recognition, 3260-3269.

Silberman, N., Hoiem, D., Kohli, P. and Fergus, R., 2012. Indoor segmentation and support inference from rgbd images. Proc. Europ. Conf. on Computer Vision, 746-760.

Simonyan, K. and Zisserman, A., 2014. Very Deep Convolutional Networks for Large-Scale Image Recognition. arXiv preprint arXiv: 1409.1556.

Wang, C., Buenaposada, J. M., Zhu, R. and Lucey, S., 2018. Learning depth from monocular videos using direct methods. Proc. IEEE Conf. on Computer Vision and Pattern Recognition, 2022-2030.

Wenzel, K., Rothermel, M., Haala, N. and Fritsch, D., 2013. SURE - the IFP software for dense image matching. Proc. Photogrammetric Week, $59-70$.

Xie, J., Girshick, R. and Farhadi, A., 2016. Deep3D: Fully automatic 2dto-3d video conversion with deep convolutional neural networks. Proc. Europ. Conference on Computer Vision, 842-857.

Zhao, H., Gallo, O., Frosio, I. and Kautz, J., 2016. Loss functions for image restoration with neural networks. IEEE Transactions on Computational Imaging, 3(1), 47-57.

Zhou, T., Brown, M., Snavely, N. and Lowe, D. G., 2017. Unsupervised learning of depth and ego-motion from video. Proc. IEEE Conf. on Computer Vision and Pattern Recognition, 1851-1858. 